\title{
Suppression of Atrial Fibrillation by Atrial Pacing
}

\author{
Noriko Inoue, MD; Toshiyuki Ishikawa, MD; Shinichi Sumita, MD; \\ Tsukasa Kobayashi, MD; Kohei Matsushita, MD; Katsumi Matsumoto, MD; \\ Minoru Taima, MD; Miei Shimura, MD; Kazuaki Uchino, MD; \\ Kazuo Kimura, MD; Satoshi Umemura, MD
}

\begin{abstract}
Background Atrial fibrillation (AF) is a common arrhythmia in patients with an implanted pacemaker, but the role of atrial pacing in preventing $\mathrm{AF}$ is still unclear.

Methods and Results Sixty-six patients (67.8 \pm 12.1 years) were enrolled: 54 with sick sinus syndrome (SSS), 11 with atrioventricular blocks (AVB), and 1 with SSS and AVB. The prevalence of AF was investigated. In 22 patients with AF, the AF burden was estimated under "back-up pacing" (40-50 beats/min), then under "atrial pacing" (60-85 beats/min). The prevalence of AF in the SSS group tended to be higher than that in the AVB group (48.1\% vs $18.2 \%, \mathrm{p}=0.06)$. The AF burden in patients with a percentage of atrial pacing (\% atrial pacing) $<50 \%$ was significantly greater than that in patients with \% atrial pacing $\geq 50 \%(12.5 \pm 21.1 \%$ vs $4.2 \pm 10.3 \%, \mathrm{p}<0.05)$. AF disappeared immediately after "atrial pacing" in 4 patients (18.2\%). In 9 patients (40.9\%), the AF burden

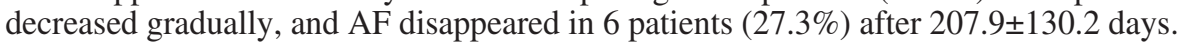

Conclusion The prevalence of AF may be higher in patients with SSS than in those with AVB. Atrial pacing has a preventive effect on $\mathrm{AF}$, and the effect of atrial pacing is not always immediate but is progressive in some patients. (Circ J 2006; 70: 1398-1401)
\end{abstract}

Key Words: Atrial fibrillation; Pacemaker; Pacing

$\mathbf{P}$ aroxysmal atrial fibrillation (AF) is a common arrhythmia in patients with an implanted pacemaker. Previous studies have suggested that atrial pacing in patients with sick sinus syndrome (SSS) is associated with a lower frequency of AF. Some studies have investigated the long-term effect of atrial pacing in AF suppression. Most of these studies compared physiological pacing with non-physiological pacing $!^{1-3}$ The role of atrial pacing itself in preventing AF still remains unclear. For patients with an implanted pacemaker, atrial tachyarrhythmia detection with the implanted pacemaker is useful. However, atrial far-field oversensing from the ventricle is one of the greatest problems in the automatic interpretation of memory functions in DDD pacemakers. In many studies of AF suppression using pacing, far-field oversensing was not excluded. We examined the prevalence of AF in SSS and atrioventricular block (AVB), and the beneficial effect of atrial pacing itself, compared with "back-up pacing", not with ventricular pacing. Moreover, we investigated the long-term effect of continued atrial pacing in AF suppression.

\section{Methods}

\section{Patients}

Between May 1998 and July 2002, 67 patients who underwent implantation of a dual-chamber pacemaker (Vitatron, Selection ${ }^{\circledR}$, Dieren, NL) for bradycardia were

(Received April 17, 2006; revised manuscript received August 4, 2006; accepted August 24, 2006)

Department of Medical Science and Cardiorenal Medicine Yokohama City University Graduate School of Medicine, Yokohama, Japan Mailing address: Noriko Inoue, MD, Department of Medical Science and Cardiorenal Medicine Yokohama City University Graduate School of Medicine, 3-9 Fukuura, Kanazawa-ku, Yokohama 236-0004, Japan.

E-mail: norikok@yokohama-cu.ac.jp recruited. Far-field $\mathrm{R}$ wave oversensing was observed in 7 patients. In 6 patients, it could be avoided by changing the pacemaker's programming. They were subsequently included in the present study. However, one patient, in whom far-field oversensing could not be avoided, was excluded. Thus, 66 patients, of which 33 were male, with a mean age of $67.8 \pm 12.1$ years, were enrolled after giving informed consent. The indication for pacing was SSS in 54 patients, AVB in 11 and SSS with AVB in one. Although some patients were taking antiarrhythmic agents during the study period, the drugs all patients were already receiving were left unchanged.

\section{Study Protocol}

We investigated the prevalence of AF in all patients during the 28 days immediately after implantation. Twentytwo (21 with SSS, 1 with AVB) had paroxysmal AF. The pacemakers were programmed for "back-up pacing" (4050 beats $/ \mathrm{min}$ ) at first. Subsequently, the pacemakers were programmed for "atrial pacing" (60-85 beats $/ \mathrm{min})$. The follow-up period for each program was 3 months. The AF burden (\%) was estimated under "back-up pacing" (4050 beats/min) and "atrial pacing" ( $60-85$ beats/min). Afterwards, all patients were left with their pacemaker programmed to "atrial pacing". The AF burden (\%) was followed continuously in these patients. The follow-up time was more than 15 months. The AF burden (\%) was calculated as the proportion of the total duration of $\mathrm{AF}$ detected during the follow-up period.

\section{Statistical Analysis}

The values are given as mean \pm standard deviation. Data were compared using Student's t-test. A p value $<0.05$ was considered significant. 

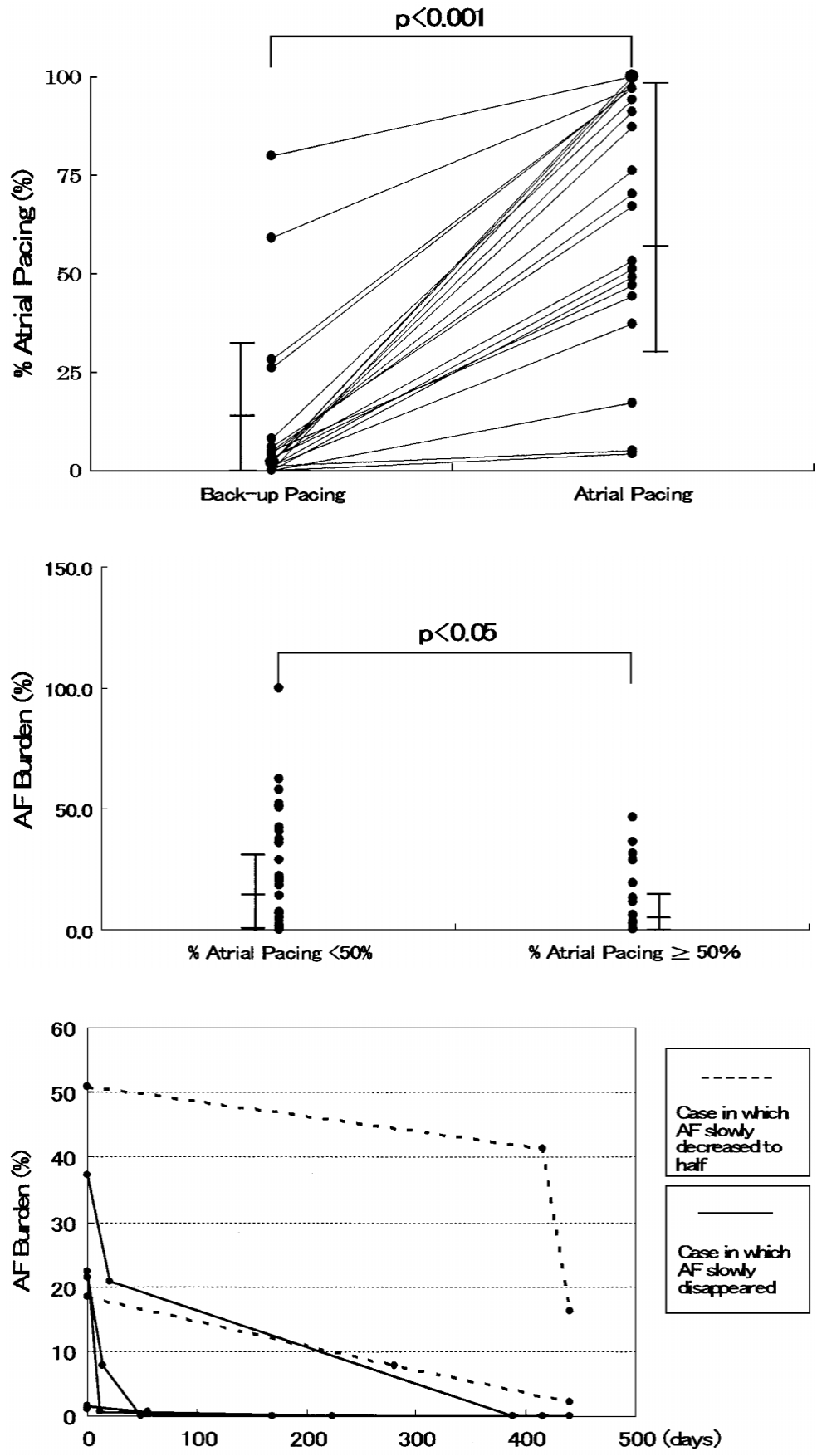

Fig 1. Percentage atrial pacing under "atrial pacing" was significantly higher than that under "back-up pacing" $(67.3 \pm 32.1 \%$ vs $10.8 \pm 20.7 \%$, p $<0.001)$.
Fig 2. Atrial fibrillation (AF) burden in patients with $\%$ atrial pacing $<50 \%$ was significantly greater than that in patients with $\%$ atrial pacing $\geq 50 \%(12.5 \pm 21.1 \%$ vs $4.2 \pm 10.3 \%, \mathrm{p}<0.05)$.
Fig 3. Time course changes in atrial fibrillation (AF) burden after physiological pacing. In 4 cases, AF slowly decreased and disappeared at a maximum of 390 days after starting atrial pacing. In 2 cases, AF did not disappear; however, the AF burden (\%) continued to decrease 450 days after starting atrial pacing.

\section{Results}

Prevalence of $A F$

The prevalence of AF during the 28 days after enrollment tended to be higher in the SSS group than in the AVB group $(48.1 \%(26 / 54)$ vs $18.2 \%(2 / 11), p=0.06)$.

\section{AF Burden}

The pacing \% under "atrial pacing" was significantly higher than that under "back-up pacing" $(67.3 \pm 32.1 \%$ vs $10.8 \pm 20.7 \%, \mathrm{p}<0.001$ ) (Fig 1). Of the patients who had AF, the $\mathrm{AF}$ burden in those with an atrial pacing (\% atrial pacing) percentage of $<50 \%$ was significantly greater than that in those with $\%$ atrial pacing $\geq 50 \%(12.5 \pm 21.1 \%$ vs $4.2 \pm 10.3 \%, \mathrm{p}<0.05)($ Fig 2$)$.
AF Suppression by Atrial Pacing

AF disappeared immediately after "atrial pacing" in 4 of the 22 patients $(18.2 \%)$. In 9 patients $(40.9 \%)$, the AF burden decreased to less than $50 \%$ in $174.3 \pm 155.3$ days, and AF completely disappeared in 6 of them $(27.3 \%)$ in $207.9 \pm 130.2$ days.

\section{Long-Term Effect of Atrial Pacing}

In 4 cases, the AF burden slowly decreased and disappeared, the longest interval after which AF disappeared was 390 days from starting atrial pacing. In 2 cases, AF did not disappear, but the AF burden (\%) decreased by less than half and still continued to decrease 450 days after starting atrial pacing (Fig 3). 


\section{Discussion}

The first major finding in the present study was that the prevalence of AF tended to be higher in SSS patients than in AVB patients. Second, atrial pacing in patients with a $\%$ atrial pacing of more than $50 \%$ had a preventive effect on AF. The preventive effect of atrial pacing on AF did not always seem to be immediate; it was progressive in some patients.

In present study, AF tended to be more prevalent with SSS patients than AVB patients. A previous report on the histological findings of SSS demonstrated that the atrium was likely to consist of fibrosis or fatty infiltration? A recent study has demonstrated that atrial fibrosis can provide a pathophysiologic substrate for AF, which results in increased conduction heterogeneity associated with the inducibility of AF6 Therefore, it is of concern that SSS patients with fibrosis in the atrium have substrate for AF. It is likely to induce the occurrence and maintenance of AF. A previous study showed that the prevalence of supraventricular arrhythmias was higher, but not significantly, in AVB than in sinus node disease and sinus node disease with $\mathrm{AVB} ;{ }^{7}$ however, far-field oversensing was not excluded in the previous study. When we interpret the Holter memory in an implanted pacemaker, we should distinguish far-field oversensing from atrial high rate episodes. Some studies have reported the incidence of far-field oversensing. It was reported that the incidence of inappropriate mode switching caused by atrial preventicular oversensing related to near-field $\mathrm{P}$ wave or far-field $\mathrm{R}$ wave oversensing in a dual chamber pacemaker is up to $10 \% 4,8$ Therefore, atrial far-field oversensing from the ventricle is one of the greatest problems in the evaluation of atrial pacing for the prevention of AF. We examined the prevalence of AF in SSS and AVB, after excluding far-field oversensing.

High atrial pacing $(\%)$ was associated with a reduction in the AF burden (\%). Previous studies suggested that atrial pacing may have a preventive effect on AF. However, most of them compared physiological pacing with non-physiological pacing. Physiological pacing can be performed using 2 methods; single chamber atrial pacing and dual chamber pacing. One study showed that single chamber atrial pacing was associated with a lower frequency of $\mathrm{AF}$, though not significant, than single chamber ventricular pacing in patients who had SSS for 3 years! Moreover, a study with extended follow-up period of up to 8 years showed a beneficial effect from atrial pacing on the incidence of AF? In a prospective randomized study that examined the risk of AF, dual chamber pacing significantly decreased the risk of AF compared to single chamber ventricular pacing in patients with sinus node dysfunction? In those studies, the effect of atrial or dual chamber pacing was often compared to that of ventricular pacing. However, it has been reported that ventricular pacing is associated with cardiac function abnormalities and enlargement of the left atrium. Ventricular pacing might cause a proarrythmic effect due to impaired ventricular function and atrial stretch from valvular regurgitation.10-12 Therefore, the effect of atrial pacing alone for preventing AF in patients with symptomatic bradycardia still remains unclear. It is important that we investigate the preventive effect of atrial pacing compared with no pacing on the occurrence or frequency of AF. Some previous studies examined whether physiological pacing would prevent AF when compared with no pacing 13 In one study, it was demonstrated that physiological pacing prevented AF compared to no pacing. 14 On the other hand, another study failed to demonstrate that physiological pacing prevented AF compared to no pacing in patients with frequent paroxysmal AF. However, there was no symptomatic bradycardia in the study population. 15 The $\%$ atrial pacing may be low in such a situation. Physiological pacing cannot be performed sufficiently using the usual pacemaker settings with a base rate of 60 beats $/ \mathrm{min}$ in patients without bradycardia. The role of physiological pacing in preventing AF may not be significant in patients without symptomatic bradycardia. In such a study population, the $\%$ atrial pacing should be low. On the other hand, in a study examining the benefit of atrial overdrive pacing in the prevention of AF in patients with SSS, there was no significant benefit of atrial overdrive pacing when compared to atrial pacing with a base rate of 60 beats $/ \mathrm{min} \cdot{ }^{16}$ However, there was no significant difference in the $\%$ atrial pacing between the 2 groups. The $\%$ atrial pacing with a base rate of 60 beats/min was $60 \pm 26 \%$ and that with atrial overdrive pacing was $72 \pm 7 \%$. We consider that the total duration of atrial pacing is important in obtaining a beneficial effect in the prevention of AF. We investigated the effect of atrial pacing, not compared with ventricular pacing, in relation to $\%$ atrial pacing, and there was a significant difference between AF burden with \% atrial pacing $<50 \%$ and that with $\%$ atrial pacing $\geq 50 \%$. Moreover, we investigated the long-term effect of continued atrial pacing for AF suppression. No study has investigated the disappearance of AF using atrial pacing in the long term. Our findings suggest that atrial pacing is effective in AF suppression, and the benefits can appear immediately or gradually. Some recent clinical and experimental studies have shown that AF alters the electrical and mechanical remodeling of the atrium, which results in the maintenance and recurrence of AF ${ }^{17,18}$ In the study of an experimental model of AF, it was reported that reverse remodeling of atrial electrical properties following conversion to AF occurred ${ }^{19}$ In humans, the AF cycle length as a measure of atrial refractoriness and the shortest coupling interval of atrial premature beats as a separate measure of atrial refractoriness were examined before the initial cardioversion and at the time of the first $\mathrm{AF}$ recurrence to investigate the reversibility of atrial electrical remodeling. ${ }^{20}$ It was shown that the changes in atrial electrophysiology associated with chronic AF were reversible after cardioversion and that the extent of this reversal was dependent on the duration of sinus rhythm after cardioversion. There are some reports about atrial mechanical remodeling. The maintenance sinus rhythm using cardioversion with implantable atrial defibrillator reduced the total burden of AF and the left atrial size. That is, it was shown that maintaining sinus rhythm caused a reversed atrial mechanical remodeling in the patients with persistent $\mathrm{AF}^{21}$ Furthermore, in another study, it was reported that the recovery of left atrial mechanical function was related to the duration of AF before chemical or direct current cardioversion.22 From the results of these reports, prompt cardioversion of AF reduces AF burden and reverses atrial remodeling, and prevents subsequent AF recurrence. In present study, AF disappeared in the long term after the implantation of pacemaker in some patients. The mechanism of the progressive role in the suppression of $\mathrm{AF}$ using atrial pacing may be similar to the mechanism of maintaining of sinus rhythm using repetitious cardioversion. The maintenance of sinus rhythm using atrial pacing may also cause reverse atrial remodeling of the electrophysiological and mechani- 
cal properties, and result in reduction of AF burden.

In conclusion, the prevalence of AF is higher in SSS patients than in AVB patients. Atrial pacing has a preventive effect on AF, and the preventive effect is not always immediate but is progressive in some patients.

\section{References}

1. Andersen HR, Thuesen L, Bagger JP, Vesterlund T, Thomsen PE Prospective randomised trial of atrial versus ventricular pacing in sick-sinus syndrome. Lancet 1994; 344: 1523 - 1528

2. Link MS, Estes NA 3rd, Griffin JJ, Wang PJ, Maloney JD, Kirchhoffer JB, et al. Complications of dual chamber pacemaker implantation in the elderly: Pacemaker Selection in the Elderly (PASE) Investigators. J Interv Card Electrophysiol 1998; 2: 175179.

3. Lamas GA, Lee KL, Sweeney MO, Silverman R, Leon A, Yee R, et al; Mode Selection Trial in Sinus-Node Dysfunction. Ventricular pacing or dual-chamber pacing for sinus-node dysfunction. $N$ Engl $J$ Med 2002; 346: 1854-1862.

4. Kolb C, Aratma S, Zrenner B, Schmitt C. Preventricular far-field sensing in the atrial channel of dual chamber pacemakers: An occasional cause of inappropriate mode switch. J Interv Card Electrophysiol 2004; 10: 231-235.

5. Evans R, Shaw DB. Pathological studies in sinoatrial disorder (sick sinus syndrome). Br Heart J 1977; 39: 778 - 786 .

6. Li D, Fareh S, Leung TK, Nattel S. Promotion of atrial fibrillation by heart failure in dogs: Atrial remodeling of a different sort. Circulation 1999; 100: 87-95.

7. Defaye P, Dournaux F, Mouton E. Prevalence of supraventricular arrhythmias from the automated analysis of data stored in the DDD pacemakers of 617 patients: The AIDA study: The AIDA Multicenter Study Group: Automatic Interpretation for Diagnosis Assistance. Pacing Clin Electrophysiol 1998; 21: 250-255.

8. Bordacher P, Cazeau S, Graindorg L, Ritter P. Impact and prevention of far-field sensing in fallback mode switches. Pacing Clin Electrophysiol 2003; 26: 206-209.

9. Andersen HR, Nielsen JC, Thomsen PE, Thuesen L, Mortensen PT, Vesterlund T, et al. Long-term follow-up of patients from a randomised trial of atrial versus ventricular pacing for sick-sinus syndrome. Lancet 1997; 350: 1210-1216.

10. Lee MA, Dae MW, Langberg JJ, Griffin JC, Chin MC, Finkbeiner WE, et al. Effects of long-term right ventricular apical pacing on left ventricular perfusion, innervation, function and histology. J Am Coll
Cardiol 1994; 24: 225-232.

11. Gillis AM. Pacing to prevent atrial fibrillation. Cardiol Clin 2000; 18: $25-36$.

12. Nielsen JC, Andersen HR, Thomsen PE, Thuesen L, Mortensen PT, Vesterlund T, et al. Heart failure and echocardiographic changes during long-term follow-up of patients with sick sinus syndrome randomized to single-chamber atrial or ventricular pacing. Circulation 1998; 97: 987-995.

13. Enjoji Y, Sugi K, Noro M, Nakae T, Tezuka N, Takami M, et al. Evaluation of bi-atrial pacing and single site right atrial pacing for the prevention of atrial fibrillation. Circ J 2002; 66: 70-74.

14. Gillis AM, Wyse DG, Connolly SJ, Dubuc M, Philippon F, Yee R, et al. Atrial pacing periablation for prevention of paroxysmal atrial fibrillation. Circulation 1999; 99: 2553-2558.

15. Wiberg S, Lonnerholm S, Jensen SM, Blomstrom P, Ringqvist I, Blomstrom-Lundqvist C. Effect of right atrial overdrive pacing in the prevention of symptomatic paroxysmal atrial fibrillation: A multicenter randomized study, the PAF-PACE study. Pacing Clin Electrophysiol 2003; 26: $1841-1848$.

16. Levy T, Walker S, Rex S, Paul V. Does atrial overdrive pacing prevent paroxysmal atrial fibrillation in paced patients? Int J Cardiol 2000; 75: 91-97.

17. Komatsu T, Sato Y, Tachibana H, Nakamura M, Horiuchi D, Okumura K. Randomized crossover study of the long-term effects of pilsicainide and cibenzoline in preventing recurrence of symptomatic paroxysmal atrial fibrillation: Influence of the duration of arrhythmia before therapy. Circ J 2006; 70: 667-672.

18. Okumura Y, Watanabe I, Nakai T, Sugimura H, Hashimoto K, Masaki R, et al. Recurrence of atrial fibrillation after internal cardioversion of persistent atrial fibrillation: Prognostic importance of electrophysiologic parameters. Circ J 2005; 69: 1514-1520.

19. Lee SH, Lin FY, Yu WC, Cheng JJ, Kuan P, Hung CR, et al. Regional differences in the recovery course of tachycardia-induced changes of atrial electrophysiological properties. Circulation 1999; 99: $1255-1264$.

20. Hobbs WJ, Fynn S, Todd DM, Wolfson P, Galloway M, Garratt CJ. Reversal of atrial electrical remodeling after cardioversion of persistent atrial fibrillation in humans. Circulation 2000; 101: 1145-1151.

21. Tse HF, Lau CP, Yu CM, Lee KL, Michaud GF, Knight BP, et al. Effect of the implantable atrial defibrillator on the natural history of atrial fibrillation. J Cardiovasc Electrophysiol 1999; 10: 1200 1209.

22. Manning WJ, Silverman DI, Katz SE, Riley MF, Come PC, Doherty $\mathrm{RM}$, et al. Impaired left atrial mechanical function after cardioversion: Relation to the duration of atrial fibrillation. J Am Coll Cardiol 1994; 23: 1535-1540. 